\title{
Encoding of kinesthetic extent information
}

\author{
ERIC A. ROY \\ Department of Psychology \\ and \\ GORDON L. DIEWERT \\ Department of Kinesiology \\ University of Waterloo, Waterloo, Ontario, Canada N2L $9 G 1$
}

\begin{abstract}
The difference in codability of kinesthetic extent cues for experimenter-determined vs. subject-determined standards was investigated. The task involved moving a slide along a linear track a distance of one-half the total distance of the track. This distance (the standard) was then reproduced. During the presentation of the standard, reaction time to an auditory probe was recorded. One group of subjects determined their own standard (active condition), while the other group moved the slide to a stop located at the standard distance (constrained condition). All subjects were told that the standard was one-half the total distance. A more active encoding process was hypothesized to occur in the active condition which would be reflected in increased reaction time to the probe. The results did not support the attention hypothesis, in that probe reaction times were not significantly different for the two groups. Rather, the data suggested that the important variable in determining the codability of extent cues was the availability of a strategy and not whether the experimenter or the subject determined the standard.
\end{abstract}

The codability of kinesthetic information has been of interest to psychologists for a long time (e.g.) Leuba, 1909; Woodworth, 1899). However, not until the last two decades have the sensory cues subserving kinesthesis been isolated and investigated in detail (e.g., Bahrick, 1957; Marteniuk \& Roy, 1972; Posner, 1967). Two spatial cues involved in kinesthesis have received special attention: the extent or distance of movement of a limb and the spatial location of the limb. Extent of movement refers to the distance through which a limb is moved, while location refers to the position in space the limb occupies. In general, the methodology which has been used to investigate these cues is a variation of the psychophysical method of average error in which the subject is presented a standard movement (C) by moving a slide with his limb (usually his arm) from a starting position to a terminal position and back. He is then required to reproduce $C$.

Using this method, it is possible to isolate the cues of extent and position so that they can be examined independently. If the subject reproduces $C$ as it was presented, he has both location and extent information about $C$. In order to isolate these two cues, it is necessary to change the starting position from that used in $C$ when the subject is asked to reproduce $\mathrm{C}$. The ability of the subject to remember

Appreciation is extended to Dr. G. E. MacKinnon for helpful suggestions. Requests for reprints should be sent to Eric Roy, Department of Psychology, University of Waterloo, Waterloo, Ontario, Canada N2L 3G1. the extent of $\mathrm{C}$ is examined by having him reproduce only the extent of $\mathrm{C}$ from the new starting location. In this case, location cues are unreliable. Alternately, location information about $\mathrm{C}$ can be examined by having the subject reproduce the position his arm was in at the terminal position of $\mathrm{C}$ from the new starting location. In this case, extent cues are unreliable.

A study by Posner (1967) was one of the first to isolate the cues of extent and location and examine their retention characteristics independently. He examined the retention of kinesthetic location and extent cues with and without vision following retention intervals of 0 to $20 \mathrm{sec}$. Further, the 20 -sec retention interval was either unfilled (in which the subject could "rehearse" $C$ ) or filled, (in which the subject was required to perform a digit classification task). The results indicated that when vision was present and the retention interval was unfilled, both extent and location information were retained over the 20 -sec. When the interval was filled, however, a significant $(p<.05)$ amount of forgetting occurred. When vision was not present, a significant amount of forgetting occurred over the 20 -sec interval regardless of whether it was tilled or unfilled. Further, distance information, in general, was retained significantly less accurately than location information. These results showed that, when vision was removed, the kinesthetic cues were not retained regardless of whether the subject had an opportunity to rehearse. This suggested that purely kinesthetic cues did not acquire access to central processing as did the same cues when they were coded visually. Consequently. 
Posner (1967) suggested that kinesthetic cues were not codable.

Laabs (1973) reexamined the cues of extent and location studied by Posner (1967). He examined the retention of these cues without vision over a 12 -sec retention interval which was either unfilled or filled with a counting-backward task. The results indicated that after immediate reproduction, extent and location were equally well retained and both showed forgetting over the 12-sec interval when it was filled. However, when the interval was unfilled only the extent condition showed significant forgetting. These results suggested that the kinesthetic cues of extent and location had different encoding properties. As forgetting occurred only for extent cues over an unfilled interval, Laabs' study suggested that only extent cues are not codable.

Recently, Marteniuk (1973) found that both extent and location cues were codable: forgetting occurred for both cues over a filled 20 -sec interval; no forgetting for either cue was observed over a comparable unfilled interval. The conflicting findings of Labs (1973) and Marteniuk (1973) regarding the codability of kinesthetic extent information is enigmatic at first. However, closer examination of the methods used by these investigators suggests a reason for the discrepancy. Laabs (1973), in presenting C, had the subject move a slide with his arm between two metal stops, thus defining the $\mathrm{C}$ for the subject. Marteniuk (1973), on the other hand, had the subject make his own $C$. The subject moved his arm through a total distance (T) and back; he was then told to move a distance that was some fraction of $\mathrm{T}$ (this movement represented the $\mathrm{C}$ ), and then required to reproduce either the end location or the extent of $\mathrm{C}$ from a new starting location. The fundamental difference between the two conditions was whether the experimenter or the subject determined $C$. When the subject determined his own $\mathrm{C}$, it would appear that extent information was codable (e.g., Marteniuk, 1973), whle when $C$ was prescribed to the subject by the experimenter, extent information was not codable (e.g., Laabs, 1973).

The results of a study by Jones (1974) provides support for the foregoing argument. Jones examined the retention of extent cues under three modes of presenting C: passive, constrained, and active. In the passive and constrained conditions, stops were used to define $C$ : in the passive condition, the experimenter moved the subject's arm between the two stops, while in the constrained condition, the subject moved his own arm. In the active condition, no stops were used to define $C$; rather, the subject was instructed to make any movement he wished, and this movement defined C. In all conditions, the original starting position used for the $C$ was changed and the subject reproduced the extent of $\mathrm{C}$ from the new starting location. These conditions were examined at a 0 - and 15 -sec retention interval which was either filled or unfilled. The results showed that the active condition resulted in significantly less forgetting than the other two conditions at immediate reproduction and after an untilled $15-\mathrm{sec}$ interval. When the interval was filled, however, all groups exhibited a similar degree of forgetting.

Clearly, it appears that when the subject is allowed to define his own C (e.g., Jones, 1974; Marteniuk, 1973), extent of movement is quite codable contrary to the evidence forwarded by Laabs (1973) and Posner (1967). The question remains, however, as to why extent of movement is codable only under these restricted conditions. It is possible that when the subject actively defines $C$, a more active process of encoding extent information occurs, in that the subject is required to search for the extent of the $C$. In the constrained presentation, a more passive encoding process may ensue in which the subject relies on the experimenter to provide the extent information. This difference in encoding processes might be reflected in an increased attention demand for encoding in the active condition, which may account for the better retention in this condition. Thus, in the following experiments, an attempt was made to examine the attention demands of each method of presenting $C$.

\section{EXPERIMENT I}

\section{Method}

Subjects. Seventy-two undergraduate students from the University of Waterloo served as subjects in the study.

Apparatus. The movement apparatus consisted of a rectangular slide mounted on three 80 -in.-long stainless steel rods. Attached to the slide was a $5-\mathrm{cm}$ handle on the subject's side and a pointer on the experimenter's side to ind icate the distance moved. Under the pointer was secured a scale, calibrated in millimeters, which the experimenter used to measure the distance moved by the subject.

On the fourth stainless steel rod above the slide, a microswitch was attached to a metal sleeve. By means of the sleeve, the microswitch could be moved to any point along the rod. A small setscrew in the sleeve served to secure the microswitch in place when required. A small steel projection on the experimenter's side of the slide served to depress the switching mechanism on the microswitch when the slide moved by it.

The microswitch was connected in series to a Hueler clock timer and a white-noise generator. When the microswitch was depressed by the slide, the white-noise output from the generator was momentarily interrupted and the clock timer was activated. The timer was stopped by depressing a telegraph key which was placed on a table to the left of the movement apparatus.

A stainless steel bar, on which were four black rubber bands, ran parallel to the scale along the base of the movement apparatus. The bands served as location markers for the microswitch.

Experimental conditions. There were two conditions in the experiment: active and constrained. In the active condition, the subject defined his own $C$. The subject moved the slide a distance of $60 \mathrm{~cm}$ and back. This distance was designated the total. Then he moved the slide a distance which he considered to be half of the total. This distance constituted the $C$. Following this, he moved the slide back to a new starting location and was required to reproduce 
the extent or distance of $C$. In the constrained condition, each subject was yoked with one in the active condition on the length of C. The subject noved the slide the total distance and back. Then he mowed the slide to a stop which he was told was half the distance but which actually corresponded to the $C$ used by a matched subject in the active condition and might or might not have been exactly half. This distance to the stop was the $C$ for the subject in the constrained condition. Following the presentation of the $C$, the subject moved the slide back to a new starting position and reproduced the extent of $\mathrm{C}$.

Procedure. Before entering the testing room, the subject was blindfolded. He was led into the room and seated on a stool in front of the movement apparatus. His left arm was rested on a table to his left. and his left index finger placed on top of a telegraph key. Earphones were placed over his ears.

The experiment was divided into two phases: pretest and test. The pretest phase involved two aspects. First. 10 reaction time (RT) measures to the probe stimulus were recorded. These RTs were used as the control measures to which RTs during the test phase could be compared. Secondly, the $C$ to be used during the test phase was determined for each subject. It was necessary to establish the $\mathrm{C}$ before the test phase so that the microswitch positions could be accurately determined. The microswitch was at one of four probe positions on each trial. The first probe position was $3 \mathrm{~cm}$ from the start, while the second, third, and fourth positions were located at 1/3. $2 / 3$, and $5 / 6$ of the $C$ distance.

The test phase of the experiment involved taking RTs to the secondary stimulus and measures of accuracy in reproducing the $C$. A trial in the test phase was divided into three parts. The first part (orientation) involved moving the cursor the "total" distance. The second part (encoding) involved moving one-half the total or the $C$ and recording the RT to the probe stimulus. When the subject heard the white noise in the earphones, he moved the lever the length of $C$. While moving, the slide tripped the microswitch, which momentarily caused a cessation of white noise and began the timer. The cessation of white noise was the probe stimulus to which the subject was instructed to depress the telegraph key as fast as possible. The time between when the timer was begun and when the subject depressed the key constituted the RT measure. The subject was instructed to continue moving the slide until he reached the $C$, even though he was required to depress the telegraph key.

The third part (reproduction) involved the subject's reproducing the extent of the $C$. Following the presentation of $C$, the subject moved the slide back to a position different from the starting position used in $C$. The new starting position was one of four possible which were located at \pm 2 and $\pm 4 \mathrm{~cm}$ from the original $C$ start position. The subject then reproduced the extent of $C$. The experimenter recorded the accuracy of reproduction in terms of algebraic error to the nearest millimeter.

Each subject received 10 test trials. Eight trials were given with the probe ( 2 trials at each probe location) and 2 nonprobe or catch trials were given. Those trials were independently randomized for each subject.

\section{Results}

Reaction time. Analysis of the RT data (Figure 1) revealed a signiticant main effect of probe position, $F(3.210)=30.56, p<.01$. No other main or interaction effects were significant. A Scheffé test revealed that Probe Position 1 had significantly $(p<.01)$ longer $R T$ than the mean of the other three and thall Probe Position 4 had a longer RT than the mean of Probes 2 and 3. A Dunnett's test revealed that all the probe $\mathrm{R}$ l's were significantly longer than the control RTs. Clearly, these findings do not support the hypothesis suggested above that the attention demands for each method of presenting $\mathrm{C}$ are different.

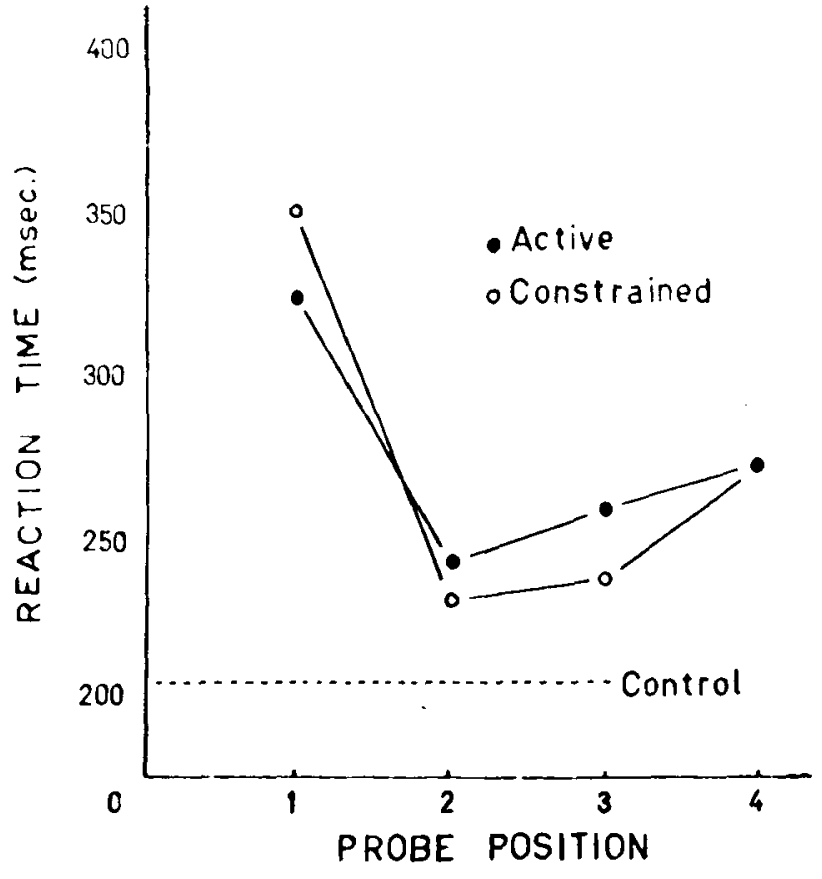

Eigure 1. Probe reaction times for each response condition.

Absolute error. The accuracy of reproduction of the $C$ for each group was calculated in terms of absolute error (AE). Five separate average reproduction accuracy measures (in terms of AE) were determined for each group and were calculated basically to assess whether the probe task, given on the encoding part of each trial, had any effect on the reproduction of the C. The first four measures, corresponding to Probe Positions 1 through 4, represented reproduction accuracy when the probe had been at the particular position during encoding. The fifth measure represented accuracy on the catch trial. An analysis of these data (see Figure 2 on following page) revealed a significant main effect of groups $[\mathrm{F}(1,70)=12.26$, $p<.01]$ and of probe positions $[F(4,280)=4.92$, $\mathrm{p}<.05]$. Also, there was a significant Group by Probe Position interaction $[\mathrm{F}(4,280)=3.19$, $\mathrm{p}<.05]$.

Subsequent analysis using the Scheffé procedure revealed that, in the active group, there were no significant differences in accuracy among any of the probe positions or between the catch and the probed trials. In the constrained group, there was a significant difference between Probe Position 1 and the mean of the other four probes. Finally, the analysis revealed that the only significant difference between the groups resulted at Probe Position 1.

\section{Discussion}

The RT data from this experiment offer no support for the notion that the difference in codability for the 


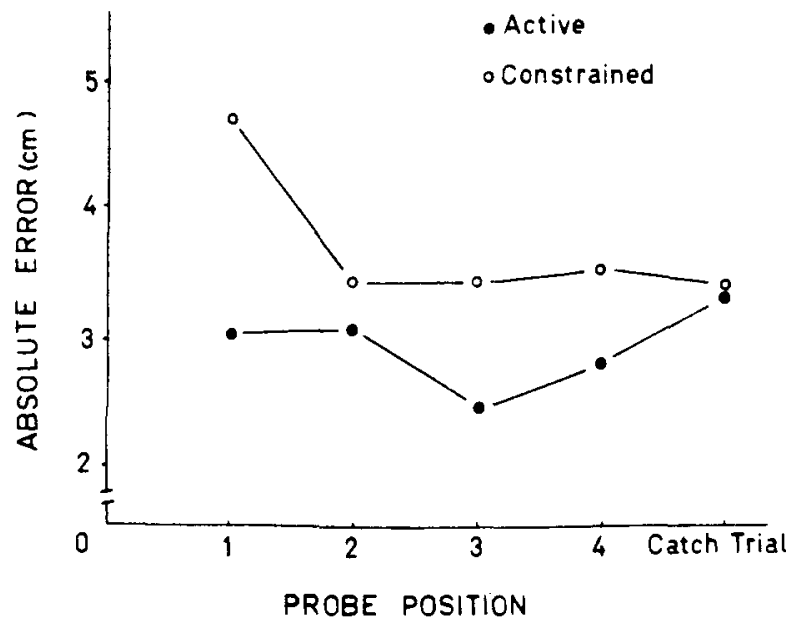

Figure 2. Accuracy of reproducing the standard for each response condition.

two methods of presenting $\mathrm{C}$ is based on a greater attention demand in the active condition. However, the usefulness of these RT data as an indicator of attention demand comes into question on two accounts. First. upon examining the AE data, it appears that. at least in the case of the constrained group. reproduction accuracy was affected by the position of the probe stimulus. suggesting that the ione. rather than simply acting as a probe, actually intluenced performance on the primary (movement) lask. Secondly, due to the small number of trials per subject, the reliability of the RT data is suspect.

While both of these considerations present formidable problems regarding the interpretation of the data. the latter seems by far the more crucial. Consequently, a second experiment was carried out in which a few subjects were run over many trials at each probe position. Using this paradigm will certainly increase the reliability of the data and, concomittantly, may reduce the apparent dependency of the probe and movement tasks.

\section{EXPERIMENT II}

\section{Method}

The apparatus, procedures, and experimental conditions were the same as in Experiment I, except that 200 trials were given to each subject: 100 control RT measures and 100 trials on the probe task. Fifty control RT measures were given before and after the probe trials. Twenty trials were given at each probe position, and 20 catch trials were given. These 100 probe trials were given in four blocks of 25 trials each. Each block of trials was constructed on the Latin square principle so that each row in the 5 by 5 square had a different combination of the five possible treatments, i.e., P1, P2, P3, P4, or catch. Four different squares were used. The same four squares were used for each subject.

Subjects. Eight students served as subjects in this experiment.

\section{Results}

Reaction time. Analysis of the RT data (Table 1) revealed a main effect of probe position $[F(3.18)=$ $27.92, \mathrm{p}<.001]$. No other effects were signiticant. Subsequent tests using the Scheffé procedure revealed that RT at Probe Position 1 was significantly greater than the other three; Probe Position 4 was signiticantly longer than Probe Positions 2 and 3. A Dunnett's test revealed that each probe RT was significantly greater than the control.

Absolute error. Analysis of the reproduction accuracy (Table 1) revealed no significant effects.

\section{Discussion}

The RT data generally corroborate those of the first experiment: the latencies to the probe stimulus are increased at either end of the movement. while all the latencies are greater than the control, and no main effect of groups was present, again lending no support to the attention hypothesis entertained at the outset.

The AE data of this experiment, in general, do not concur with those of Experiment $I$ in that none of the effects were significant. In Experiment I, recall that the group main effect in terms of AE was all centered around the group differences at Probe Position 1. This interpretation was supported by the Group by Probe Position interation.This finding led to the supposition that the probe task and the movement task were not really independent, thus making the probe task suspect as a true measure of attention. In this experiment, however, there was no interaction or an effect of probe positions, suggesting that the existence of these effects in Experiment I may have been an artifact due to the few trials given to each subject. Since these effects were not present, more confidence can be placed in the interpretation of the RT data.

\section{GENERAL DISCUSSION}

The RT data from these experiments offer no support whatsoever for the notion that the difference

Table 1

Reaction Time and Reproduction Accuracy for Each Response Condition

\begin{tabular}{|c|c|c|c|c|c|c|c|c|c|c|}
\hline \multirow{3}{*}{$\begin{array}{l}\text { Movement } \\
\text { Condition } \\
\text { Active }\end{array}$} & \multicolumn{10}{|c|}{ Probe Position } \\
\hline & \multicolumn{2}{|c|}{1} & \multicolumn{2}{|c|}{2} & \multicolumn{2}{|c|}{3} & \multicolumn{2}{|c|}{4} & \multicolumn{2}{|c|}{ Control } \\
\hline & 300.8 & $(3.9)^{*}$ & 264.2 & $(3.9)$ & 252.7 & $(3.5)$ & 288.1 & $(4.1)$ & 204.1 & (3.6) \\
\hline Constrained & 330.7 & (3.5) & 257.5 & (3.3) & 245.3 & (3.5) & 272.8 & (3.1) & 211.1 & (3.2) \\
\hline
\end{tabular}

*Values in parentheses represent $A E$. 
in codability for the two methods of presenting $C$ is based on a greater attention demand in the active condition. While these results are puzzling, two explanations can be entertained. First, it is possible that the subjects were simply switching attention from the lever-positioning task to the probe RT task so that they could maximize performance on the RT task. It seems rather improbable, however, that such shifts in attention could have occurred as the probe was moved to different positions randomly from trial to trial. Nevertheless, the contingencies associated with each probe position were different. In fact, given that the probe had not occurred in the first three positions, the probability was at its highest $(.50)$ that it would occur in the fourth, thus the lowest probe RT might be expected at the last position. Examination of Figure 1 and Table 1 shows that this did not occur. Rather, there was a marked increase in RT in this position. In general, then, an attentional shift explanation appears untenable.

The second and perhaps more parsimonious explanation is that the two groups were using the same control mechanism. It may be remembered that the groups were equated in that they were provided with the same strategy of moving the total distance followed by moving half the distance defined as the $\mathrm{C}$. The only difference betwen the groups was whether the experimenter (constrained condition) or the subject (active condition) determined when the movement would end. The similarity in strategy given to the two groups may have resulted in their employing the same controlling mechanism. That is, the searching behavior hypothesized to occur under the active condition may have been operating also under the constrained condition.

In this regard, it is interesting to examine the probe R'T data reported by Posner and Keele (1969). In this study, RT to a probe was examined both during a visually guided movement to a target and during a blind movement to a stop. In both movements, when the probe was near the beginning or the end of the movement, RT was elevated above that of the control. When the probe was in the middle of the movement, however, the probe RT was significantly higher than the control only in the visually guided condition. This suggested that only initiation and termination of movement required attention, while movement itself demanded no attention. The significance of these findings to the interpretation of this experiment is realized upon examining the $\mathrm{RT}$ data for the constrained condition. As in Posner's blind movement condition, subjects in the constrained condition were required to move to a stop. Thus. one would expect, based on Posner's blind movement RT data, that the probe R'T would only be greater than the control in extreme probe positions, since movement itself was show n to require no attention (Posner \& Keele, 1969).
These data clearly do not conform to this prediction in that all the probe RTs were greater than the control; rather, they are most reminiscent of the results afforded under the visually guided movement condition. Consequently, one is led to the conclusion that the subjects were not simply moving to the stop, but rather were processing some type of information during the movement. Just as subjects using the visually guided movement were monitoring visual cues during movement (Posner \& Keele, 1969), the subjects in this experiment appeared to be monitoring information in order to encode the extent of movement.

That similar strategies were used in both conditions is supported by the AE data (Figure 2 and Table 1 ) in that there were no meaningful diffgerences between the groups in terms of the error in reproduction. This finding is in marked contrast to those reported by Jones $(1972,1974)$, who found a significant difference in AE between the active and constrained groups. This discrepancy with the Jones data can be explained if one considers the role of strategy mentioned above.

Jones $(1972,1974)$ failed to control for encoding strategy in his active and constrained conditions. Since subjects in his active condition moved to a distance of their choice while subjects in his constrained condition simply moved to a stop, there would have been a large difference in strategy between the two conditions. That is, to the active subjects, the to-be-remembered movement had some meaning in that they themselves had decided how far to move the cursor; to the constrained subjects, however, the movement had little relevance, since the experimenter, in using the stop. effectively decided how far they were to move. Consequently, the strategy in encoding the information would have been markedly different. These subjects would not be "searching" for a distance as in the active condition. Unlike the active condition, they had no control over when to terminate their response.

In this study, however, encoding strategy was equated in that both groups knew that the $C$ was half the total. In both groups, then, the subjects had some information about when to terminate their response. Consequently, as alluded to above, both groups appeared to be monitoring the same information during encoding, suggesting that they both may have been "searching" for the C.

In summary, these results suggest that a major difference in terms of codability between the active and constrained conditions observed in the Jones $(1972,1974)$ experiments may have been due to an absence of strategy in the constrained condition since, in this study (e.g., Jones, 1974), where strategy was given in the constrained condition, reproduction accuracy comparable to that in the active condition accrued. 
This interpretation suggests, therefore, that the codability of extent information is not related to whether the experimenter or the subject determines the $C$ as Jones (1974) would have us believe. Rather, codability seems to be related to whether the subject has prior information about the to-be-remembered movement regardless of whether he or the experimenter determines its extent.

\section{REFERENCES}

BAHRICK, H. P. An analysis of the stimulus variables influencing the proprioceptive control of movements. Psychological Review. 1957, 64, 324-328.

Jones, B. Outflow and inflow in movement duplication. Perception \& Psychophysics, 1972, 12, 95-96.

JONES, B. The role of central monitoring of efference in shortterm memory for movements. Joumal of Experimental Psychology. 1974. 102. 37-43.
LAABS, G. J. Retention characteristics of different reproduction cues in motor short-term memory. Journal of Experimental Psychology. 1973, 100, 168-177.

LEUBA. J. H. The influence of the duration and rate of arm movements upon judgement of their length. American Journal of Psychology. 1909, 20. 374-385.

MARTENiUK. R. G. Retention characteristics of motor short-term memory cues. Journal of Motor Behavior, 1973, 5, 249-259.

Marteniuk, R. G., \& Roy, E. A. The codability of kinesthetic location and distance information. Acta Psychologica. 1972. 36. $471-479$.

Posner. M. J. Characteristics of visual and kinesthetic memory codes. Journal of Experimental Psychology, 1967. 75. 103-107.

Posner. M. J., \& Keele, S. W. Attention demands of movements. Proceedings of the Seventeenth Congress of A pplied Psychology. Amsterdam: Zertlinger, 1969.

WoODworth, R. S. The accuracy of voluntary movement. Psychological Review, 1899, e(Monogr. Suppl. 2).

(Received for publication July 29, 1974; revision received February 11, 1975.) 